\title{
Diseño de interfaces y modelos de análisis y evaluación en entornos post- digitales. Casos de aplicación con recursos educativos abiertos y repositorios Interface design and analysis and evaluation models post-digital environments Application cases with open educational resources and repositories
}

\author{
> Diana Rodríguez Barros \\ FAUD UNMdP, Argentina \\ dibarros@mdo.edu.ar
}

\author{
> Stella Maris Massa \\ FI UNMdP, Argentina \\ smassa4@gmail.com
}

\begin{abstract}
We present studies and advances that link two I+D+T projects based in FADU and FI UNMdP, Argentina. These studies, started this year, have the common aim from two complementary visions, enriching an indexed and classified heritage about Open Educational Resources and repositories in postdigital environments and academic areas. This is a collaborative and interdisciplinary work with the purpose of supporting the improvement of presence, semi-presence, and distance educational process, and helping free and equal access to different available educational resources. At this state, in particular, we are interested in contributing to the improvement of REA interface designs and repositories through providing heuristic lists that join later an analysis and evaluation interface model.
\end{abstract}

Keywords: Diseño interfaces; Modelo análisis y evaluación; Recursos educativos abiertos; Repositorios.

\section{Introducción}

Presentamos estudios y avances que vinculan dos proyectos de Investigación, Desarrollo y Transferencia $\mathrm{I}+\mathrm{D}+\mathrm{T}$ con dirección y codirección de las autoras, radicados en la Facultad de Arquitectura, Urbanismo y Diseño y la Facultad de Ingeniería de la Universidad Nacional de Mar del Plata, Argentina. Estos estudios, iniciados en el presente año, tienen como objetivo común desde dos visiones complementarias, enriquecer un acervo clasificado e indexado sobre Recursos Educativos Abiertos y repositorios en entornos postdigitales en ámbitos académicos universitarios.

Es un trabajo colaborativo e interdisciplinario con el fin de apoyar la mejora de los procesos educativos presenciales, semipresenciales y a distancia, así como facilitar accesos libres e igualitarios a diversos tipos de recursos educativos disponibles. Por un lado, para su uso, reutilización, adaptación e intercambio. Por otro, para aportar al mejoramiento del diseño de las interfaces, entendidas a éstas como objetos técnicos y culturales del mundo cotidiano, formalizadas en espacios y superficies donde se producen operaciones de visualización, acceso, gestión, interacción e intercambio con la información.

\section{Marco conceptual}

En la sociedad actual del conocimiento y la cultura visual, observamos prácticas docentes innovadoras en algunos casos de naturaleza disruptiva, competencias híbridas en los estudiantes, así como renovación de modalidades de clases y materiales de enseńanza. (Acaso, 2013; Brown, 2013; Cobo y Moravec, 2011).
Aportan a estos escenarios el movimiento REA Recursos Educativos Abiertos (OER Open Educational Resources) para el diseño e implementación de contenidos didácticos sobre enseñanza, aprendizaje e investigación. En tanto son de dominio público, su potencial reside en la capacidad de proporcionar alternativas de mejora a las intervenciones didácticas ofreciendo acceso de naturaleza abierta a la información. Tienen antecedentes de desarrollo de software de código libre, promocionan estándares de licenciamiento diferentes a las leyes que contempla el derecho internacional, consienten su uso de forma pública y gratuita y admiten la generación de obras derivadas por otros (Smith \& Casserly, 2006; Butcher, Kanwar, Uvalic-Trumbic, 2011). Podemos considerar, sintéticamente, que están constituidos por contenidos educativos específicos sobre diferentes objetos de aprendizaje; herramientas, aplicaciones y plataformas para desarrollar, utilizar y distribuir contenidos; implementaciones de licenciamiento Creative Commons.

Estos recursos se organizan en Repositorios que, como reservorios de alta calidad, dan soporte a la gestión y difusión de los mismos (Lynch, 2013). Tales almacenamientos digitales formalizan ambientes centralizados, indexados, interconectados a la Web y vinculados generalmente a portales. Permiten que la información puedan ser incorporada, almacenada, organizada, preservada, publicada, visibilizada, consultada y distribuida. También, que pueda ser accesible y compartida libremente por la comunidad académica para, en la eventualidad, ser actualizada o ampliada.

Tales recursos y reservorios son visibilizados y accesibles desde las interfaces, ambientes de intermediación que facilitan el acceso 
desde los sitios web y los portales contenedores. (OECD, 2007). De tal forma, desde el diseño de las interfaces se mediatizan acciones en torno a estas operaciones. El buen diseño de las interfaces, en tanto práctica compleja, debería estimular la comprensión y organización de la presentación de la información a usuarios desinformados para ser asimilada y promover interacciones significativas; organizar, clasificar, catalogar e indexar sistemas de información por medio de estructuras relacionales para facilitar la recuperación y administración de información; simplificar la navegación y recorrido para conseguir resultados óptimos en la localización de la información; orientar al usuario generando interés sostenido en el tiempo; resolver la realización de actividades, control y comunicación efectiva entre usuarios, equipos, programas y redes, para producir intervenciones simples, productivas y satisfactorias; estimular grados de buen uso, satisfacción subjetiva y buenas experiencias de usuarios; relacionar niveles de acción, resultados y emociones que percibe el usuario; estimular procesos cognitivos, rendimiento y apreciación de la información en situaciones gratificantes y fructíferas para los usuarios (Rodríguez Barros, 2011).

\section{Metodología y técnicas empleadas}

La investigación tiene como objetivos, primero en relación con los REA, sistematizar procesos de producción que cumplan criterios de calidad y procedimientos de evaluación para que las secuencias didácticas devenidas resulten satisfactorias. Segundo, en relación con los Repositorios, sistematizar procesos de gestión, diseño, análisis, evaluación y preservación de las interfaces.

Actualmente, en etapa inicial de los estudios y en relación con el segundo objetivo, detectamos que existen diferentes métodos de análisis y evaluación de diseño de interfaces de Repositorios REA que varían en su rigurosidad, costos y conocimientos necesarios. Registramos como antecedentes a principios heurísticos validados (Nielsen, 2002, 2005 y Tognazzini, 2002); estándares normatizados sobre usos y calidad (ISO13407, ISO91262009, ISO/IEC9241, ISO/IECTR9126, ISO25000/SQUARE, ISO14598); disposiciones W3C sobre convenciones generales de accesibilidad y grado de conformidad a criterios ergonómicos. También destacamos estudios sobre diseño de experiencias de usuarios (Shedroff, 2008) y criterios complementarios (Carreras, 2012). Asimismo, continuamos líneas de estudio abordadas en anteriores investigaciones (Massa, 2012; Rodríguez Barros, Chiarella, Bredanini, 2008).

En esta primera etapa del estudio nos interesa detectar, precisar, cuantificar y ponderar atributos de calidad que nos permitan desarrollar un modelo de análisis y evaluación apropiado a las interfaces de Repositorios de REA. Optamos por recurrir a técnicas de ponderación heurística. La enunciación de los heurísticos que integrarán el modelo en desarrollo se basó inicialmente en la Serie ISO/IEC 9241. Está centrado en los usuarios, valora calidad en uso específicamente sobre Usabilidad en Uso y Experiencias de Usuario, y adscribe a un determinado contexto de uso. Reconocen la posibilidad de explorar, describir, comparar y valorar interfaces, con el propósito de detectar y transparentar condiciones óptimas de uso y diseño.
Nos interesa que el listado sea abierto y básico, con dimensiones de análisis adaptables y/o ampliables a las circunstancias cambiantes de contextos y usos de REA y reservorios. Por tal motivo decidimos integrar, de forma sistémica e interdisciplinaria, aportes de monodisciplinas intervinculadas al Diseño de Interfaces tales como Diseño de Información, Visualización de Información, Arquitectura de Información, Encontrabilidad, Diseño de Interacción, Usabilidad y Diseño de Experiencias de Usuario.

Planteamos tres ejes de listados de heurísticos base para el modelo, centrados según variables generales de Efectividad en uso, Eficiencia en uso y Satisfacción en Uso. Incluimos en los mismo cuestiones sobre Flexibilidad en Uso y Seguridad. Asimismo hemos considerado la influencia que ejerce el concepto de affordance (Gibson, 1989), como manera de precisar y reconocer de forma simple e intuitiva los modos de uso y de interacción. En esta dirección, reconocemos series de subvariables con sus respectivas dimensiones de análisis a manera de descriptores amplios, capaces de desmembrarse en otros descriptores más precisos y específicos.

(Ver Figura1. Esquema listado heurísticos base para el modelo de análisis y evaluación de Diseño de Interfaces REA y repositorios).

\section{Criterios heuristicos sobre Efectividad en uso}

Implicamos a la precisión e integridad con la que los usuarios alcanzan los objetivos especificados. La asociamos a la facilidad de entendimiento, la facilidad de uso y asimismo a la disposición del sistema para recordar las funcionalidades y los procedimientos junto a los grados de participación en los entornos colaborativos Web 2.0.

Sobre Facilidad de entendimiento, consideramos descriptores sobre orientación del usuario desde información objetiva básica del sitio (datos del sitio web, dirección URL, autorías y responsabilidades, contactos, fecha creación y actualizaciones, registros de propiedad intelectual); facilidad de lectura (identidad de núcleos temáticos, organización coherente de información para evitar la sobrecarga cognitiva, títulos y contenidos afines, uso apropiado de lenguajes, página inicial de presentación, ausencia de faltas ortográficas); familiaridad (consistencia de formato, uso de lenguaje simple, elementos y formas según estándares, recursos de metáforas conocidas del mundo real); legibilidad visual (jerarquía visual entre relaciones todo y parte, zonificación alta de jerarquía informativa, espacios de descanso visual, distinguibilidad de íconos, logos, botones y controles, ajuste de fuente a contexto según color, tipo y tamańo, ajuste de texto e imágenes a fondo, unidad de fondos, ajuste de visualización textual, disposición de texto visible en cualquier situación, control de desplazamientos horizontales, uso coherente de paleta de colores); control y ahorro de esfuerzo (control del estado actual y sobre acciones posibles, acciones mínimas para reducción del esfuerzo cognitivo, auto-descripción de elementos, ausencia de dificultad de entendimiento de la información, mensajes de actualización, mensajes de avisos oportunos); navegabilidad (disponibilidad y accesibilidad de recorridos internos, enlaces reconocibles y presentación de estados, facilidad de acceso en amplitud, profundidad y densidad, grado de interconexión a contenidos y acciones según enlaces y nodos, entradas y salidas, ausencia de enlaces rotos, direccionalidad precisa). 

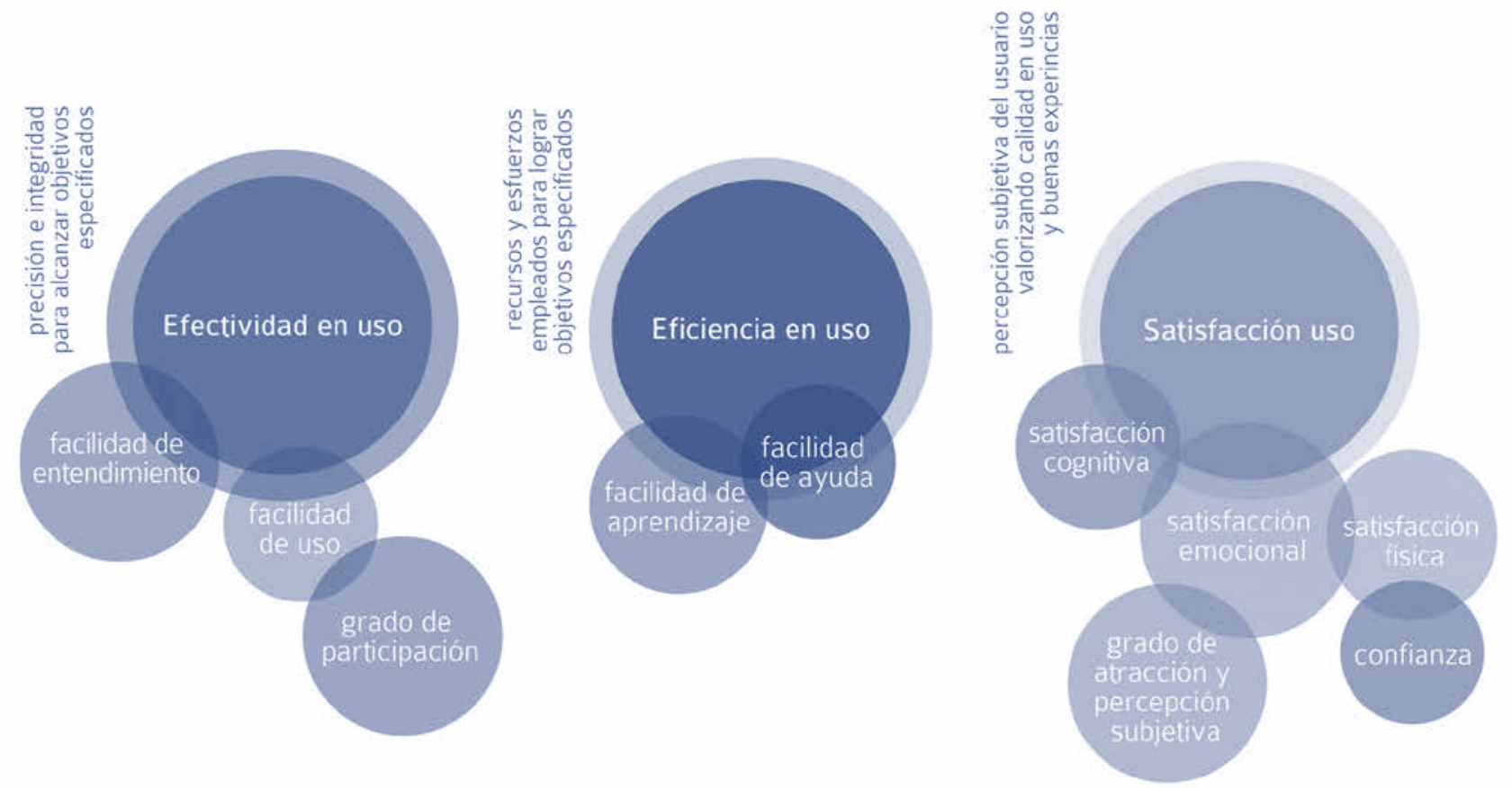

Figura 1: Esquema listado heurísticos base del modelo de análisis y evaluación Diseño de Interfaces REA y repositorios, según variables generales sobre Efectividad en uso, Eficiencia en uso y Satisfacción en Uso

Sobre Facilidad de uso, consideramos descriptores sobre compatibilidad sin alterar comportamiento y apariencia (adecuación a diversos navegadores, adecuación a velocidades de conexión, adecuación a resolución pantalla, adecuación a sistemas operativos, adecuación a aplicaciones descargables/plug-ins, soportes en varios idiomas); controlabilidad (posibilidades de búsquedas básicas y avanzadas, edición posterior, cancelación, interrupción, deshacer, rehacer, impresión, otras formas de recuperación de información, salida); adaptabilidad (a decisión del usuario desde personalización, a necesidades del usuarios); consistencia (estabilidad de comportamiento constante a enlaces, controles y etiquetas; de permanencia a enlaces y controles; de orden a enlaces y controles); gestión de los datos (validación y confirmación de datos de entrada, de datos de salida, de visibilidad de datos); gestión de errores (capacidad de prevención y de recuperación de errores, claridad de mensajes de error).

Sobre Grado de participación, consideramos descriptores sobre registros de participantes, posibilidades de intervención, modos de intervención, posibilidades de eliminación de intervenciones, información de participantes, control de restricciones, control de seguridad.

\section{Criterios heuristicos sobre Eficiencia en uso}

Implicamos a los recursos y esfuerzos empleados por los usuarios para lograr los objetivos especificados. La asociamos a la facilidad de aprendizaje en tanto supone un costo en tiempo así como al acceso a mecanismos de ayuda y facilidad del sistema para evitar una asimilación inapropiada que pueda traducirse en errores.
Sobre Facilidad de aprendizaje, consideramos descriptores sobre esfuerzo cognitivo usuario (capacidad de abordaje rápido e intuitivo, control del grado de esfuerzo mental subjetivo para obtener nivel de rendimiento adecuado, facilidad de recordar); predictibilidad (anticipación a acción de controles, enlaces, etiquetas, contenidos hipermediales); potencialidad (determinación de acciones posibles y de acciones destacadas); retroalimentación informativa (explicitación de ubicación del contexto, avances en operaciones y transacciones); rendimiento tareas (grado de completamiento, tiempo de completamiento, exactitud de tareas); limitaciones e interferencias contexto aplicación (adaptación a aplicación de habilidades del usuario, avisos de recursos requeridos a aplicación tipo descargas / plug-ing, tiempos de carga y de descargas).

Sobre Facilidad de ayuda consideramos descriptores sobre la pertinencia y completitud de ayuda en línea; posibilidad de personalizar ayudas y documentación; acceso a mapa de sitio conciso y completo; certeza y simplicidad en mensajes de asesoramiento.

\section{Criterios heurísticos sobre Satisfacción en uso}

Implicamos a la valorización del concepto de calidad en el uso que se refiere al modo en que el usuario realiza tareas específicas, en escenarios específicos, de manera gratificante, remite a los aspectos relacionados con la percepción subjetiva del usuario, ausencia de incomodidad, actitud positiva y grados de atracción al uso.

Sobre Satisfacción cognitiva consideramos descriptores sobre funcionalidades y utilidad percibida; funcionalidades y calidad de resultados.

Sobre Satisfacción emocional, consideramos descriptores sobre grado de atracción subjetiva percibida; grado de frustración percibida; grado de riesgos y de discriminaciones percibidas.

Sobre Satisfacción fisica, consideramos descriptores sobre grado de seguridad percibida. 
Sobre Grado de confianza, consideramos descriptores sobre certeza y credibilidad del sitio; desconfianza por aparición de errores; posibilidad de riesgos personales o económicos.

Sobre Grado de atracción y percepción subjetiva, consideramos descriptores sobre comprensión de estructura y organización; posibilidad de anticipación; percepción de autonomía, control y libertad; flexibilidad y simplicidad de usos; posibilidades de intervención, exploración e interacción; intuición y simplificación de aprendizajes; ausencia de desorientación y/o sobrecarga cognitiva; facilitación de legibilidad; reconocimiento del orden lógico y natural de la información; reconocimiento del diseño visual general como atractivo; coherencia del diseño visual de pantalla; ausencia de ruidos visuales y efectos superfluos; criterios de uniformidad de composiciones, colores, fondos y fuentes; utilización de imágenes atrayentes; utilización de otros recursos hipermediales acordes; posibilidades de personalizar características estéticas; posibilidad de prevenir, reconocer errores y recuperarse; acceso simple y directo a ayudas y documentación; percepción de participación placentera.

\section{Resultados}

Los enunciados de los listados presentados conforman las premisas básicas, con posibilidades de ser ampliadas, de un sistema de evaluación heurística o de inspección a implementar en esta primera etapa. Como precisa la norma ANSI-IEEE-Std/100-1984, conforman técnicas de indagación y descubrimiento aplicables en documentos con el propósito de detectar problemas tanto graves como menores, y buscar soluciones mediante reglas empíricas y métodos no rigurosos. Valoramos en este recurso el bajo costo, la ausencia de interpretación externa, las posibilidades de ser implementados por números reducidos de investigadores para analizar individual e intensivamente sitios web, describir problemas reales y/o potenciales, formalizar informes con los resultados obtenidos.

A manera de resultados provisorios, a partir de pruebas previas y aplicaciones exploratorias que hemos realizado, ponderamos que el posible modelo registra y facilita la identificación y valoración de aspectos que intervienen interactivamente con condiciones de usabilidad y componentes contextuales. También hemos observado que facilitan la detección de experiencias gratificantes de uso, factores a considerar en las fases de especificación de requisitos, diseño y evaluación de usos, así como de rendimiento y satisfacción del usuario, junto a facilitación de desarrollo de nuevas competencias. Observamos que devienen en facilitar condiciones de buena usabilidad, deriva en ventajas que involucran por un lado, disminución y optimización general de costes de diseño/producción y de aprendizaje/uso; por otro, ampliación de posibilidades de encontrabilidad, acceso, intervención y reconocimiento de condiciones óptimas de funcionalidad.

\section{Conclusiones provisorias e Implicancias}

A manera de conclusiones provisorias e implicancias, consideramos que el aporte preliminar de los listado de heurísticos al modelo, en forma amplia, aporta a viabilizar pruebas, análisis y evaluaciones para optimizar el Diseño de Interfaces. Asimismo como habilitar la interactividad, facilitar productividad, comprensión y claridad de uso de usuarios junto a disminución desorientación y sobrecarga cognitiva.
En forma restringida, aporta al reconocimiento de rutinas y estrategias sobre frecuencia / impacto / persistencia de estructuras conformantes, esquemas de visualización, organizaciones de información, modos de comunicación e interacción, junto a recursos expresivos y de lenguaje empleados.

En particular, apela a reconocer y estimular experiencias didácticas con efectos positivos que valoren estados de ánimo, percepciones y sensaciones con emociones placenteras. De forma que faciliten usos eficientes, eficaces y agradables, resignificación de información, construcción de conocimientos y estimulación de competencias híbridas en los estudiantes.

Como implicancias del estudio, nos interesa especialmente favorecer estas circunstancias en entornos virtuales postdigitales, interconectados y ubicuos que remiten a experiencias de naturaleza transmedial, colaborativas y participativas. Por lo tanto, en una segunda etapa asociaremos los listados de heurísticos a métricas de ponderación particulares considerando objetivos, interpretación, método de cálculo, fase del proceso en los que se aplican y evidencias de validez. En una tercera etapa, ya conformado el modelo, será probado y verificado en una experiencia piloto a desarrollarse hacia fines del presente año en la Plataforma educativa Moodle de la Facultad de Ingeniería UNMdP adoptando como estrategia de implementación, la combinación de tipos de evaluación de heurísticas de expertos y de test de usuarios finales o prototípicos. Consideramos al mismo como aporte amplio al mejoramiento de las condiciones de uso y diseño de las interfaces de los mismos.

\section{Agradecimiento}

El presente escrito se encuadra en estudios realizados en el marco de los proyectos de duración bianual (2014-2015) ARQ282/14 EMIDA CEAC/CIPADI FAUD SCyT UNMdP e ING412/14 GIDI ING SCyT UNMdP con dirección y codirección de las autoras.

\section{Referencias bibliográficas citadas}

Acaso, M. (2013) rEDUvolution. Hacer la revolución en la educación. Barcelona: Paidos.

Brown, T. (2013). The New Design Thinking Toolkit for Educators. En Design thinking. Thoughts by Tim Brown. Disponible http://designthinking.ideo.com/?p=894 (consultado agosto 2014)

Butcher, N., Kanwar, A., Uvalic-Trumbic, S. (2011). A based Guided to Open Educational Resources. UNESCO. Disponible http:// tinyurl.com/3fsgsrg (consultado agosto 2014)

Carreras, O. (2012). Estándares formales de usabilidad y su aplicación práctica en evaluación heurística. En Usable y Accesible. Disponible URL, http://tinyurl.com/aw46kjo (consultado agosto 2014)

OECD Centre for Educational Research and Innovation. (2007). Giving Knowledge for Free.The emergence of open educational resources. Disponible http://tinyurl.com/o655c53 (consultado agosto 2014)

Cobo, C.; Moravec, J. (2011). Aprendizaje invisible. Hacia una nueva ecología de la educación. Barcelona: Edicions de la Universitat de Barcelona. 
Lynch, C. (2013). Personal Archiving: Preserving Our Digital Heritage. En Hawkins, D. edit. Information Today: 2013. Disponible http://tinyurl.com/opcpcjl (consultado agosto 2014) Massa S.M. y Pesado P. (2012). Evaluación de la usabilidad de un Objeto de Aprendizaje por estudiantes. Revista TE\&ET Vol. 8,. Pp. 65-76.

Nielsen (2002, 2005). Ten heuristic for Usability. En useit.com: Jakob Nielsen's Website Disponible http://tinyurl.com/aruty (consultado agosto 2014)

Rodríguez Barros, D.; Chiarella, M.; Bredanini, G. (2008). Diseńo de heurísticas de usabilidad y casos de sitios web sobre gestión e industrias culturales. En Mendez, A. et al. Eds. Libro de ponencias XII Congreso SIGraDi. La Habana: Facultad de Arquitectura CUJAE. Pp. 342-346.
Rodríguez Barros, D. (2011) Capítulo 5. Redes sociales en entornos Web 2.0 y diseño de dispositivos para modelar las interacciones sociales. El caso de estudio de la Red (IA) desde la perspectiva del diseño de interfaces. En Galán, Beatriz compil. Diseño, proyecto y desarrollo. Buenos Aires: Wolkowicz Editores. Pp. 163-184.

Shedroff, N. (2008). Las emociones están en camino a la innovación significativa. En Revista Faz no 2. Pp. 8-19. Disponible http:// tinyurl.com/58j4cb (consultado agosto 2014)

Smith, M. \& Casserly, C. (2006). The Promise of Open Educational Resources. En The William and Flora Hewlett Foundation / OER. Disponible http://tinyurl.com/p7b4qfz (consultado agosto 2014) Tognazinni, B. (2002). First principles of interaction design. En Ask tog. Disponible http://tinyurl.com/2taox (consultado agosto 2014) 